\title{
A pragmatic clinicopathobiological grouping/staging system for gliomas: Proposal of the Indian TNM subcommittee on brain tumors
}

\author{
Tejpal Gupta ${ }^{1}$, Rajiv Sarin $^{1}$, Rakesh Jalalii ${ }^{1}$, Suash Sharma ${ }^{2}$, Purna Kurkure ${ }^{3}$, Atul Goel ${ }^{4}$ \\ ${ }^{1}$ Department of Radiation Oncology, ACTREC/TMH, Departments of ${ }^{2}$ Pathology and ${ }^{3}$ Pediatric Oncology, TMH, Tata Memorial \\ Centre, ${ }^{4}$ Department of ${ }^{4}$ Neuro-Surgery, KEM Hospital, Mumbai, India
}

\author{
Address for correspondence: \\ Dr. Rajiv Sarin, \\ Advanced Centre for Treatment \\ Research and Education in Cancer \\ Tata Memorial Centre, Kharghar, \\ Navi Mumbai - 410210, INDIA \\ E-mail: rsarin@actrec.gov.in
}

DOI : 10.4103/0028-3886.53261

\begin{abstract}
Background: There is no universally accepted staging system for primary brain tumors wherein prognostication is mainly based on complex composite indices. Aim: To develop a simple, pragmatic, and widely applicable grouping/staging system for gliomas, the most common primary brain tumor. Materials and Methods: An expert neurooncology panel with representation from radiation oncology, neurosurgery, pathology, radiology, and medical oncology had several rounds of discussion on issues pertinent to brain tumor staging. The trade off was between the accuracy of prognostic categorization and a pragmatic, widely applicable approach. Results and Recommendations: The Tumor-Node-Metastasis staging was considered irrelevant for gliomas that seldom metastasize to lymphatics or outside the neuraxis. Instead, a 4-point staging/grouping system is proposed, using histological grade as the main prognostic variable and at least one stage migration based on other unfavorable features such as tumor location (brainstem); age $(<5$ years for all grades, $>50$ years for high-grade, and $>40$ years for low-grade gliomas); poor neurological performance status (NPS 2-4); multicentricity and/or gliomatosis; and adverse biological parameters (proliferative index, angiogenesis markers, apoptotic index, cytogenetic abnormalities, and molecular markers). Conclusion: In absence of a grouping/staging system for primary brain tumors, prognostification is mostly based on complex composite indices. The proposed clinicopathobiological grouping/staging system for gliomas is a simple, pragmatic, and user-friendly tool with a potential to fulfill the objectives of staging classification.
\end{abstract}

Key words: Gliomas, prognosis, staging classification

\section{Introduction}

Cancer staging is a fundamental activity in oncology. The International Union Against Cancer (UICC) and American Joint Committee on Cancer (AJCC) Tumor-Node-Metastasis (TNM) system ${ }^{[1]}$ has been a benchmark for reporting the anatomic extent of disease and a major prognostic factor in predicting outcome for cancer patients for over 50 years. ${ }^{[2]}$ The major objectives of the TNM classification are to aid the clinician in planning treatment, give some indication of prognosis, assist in evaluating results of treatment, facilitate exchange of information between treatment centers, an d contribute to continuing investigations of cancer. ${ }^{[2]}$ Overall, the system is immensely useful and is a foundation of clinical decision-making and clinical practice guideline. It is indeed unfortunate that the current sixth edition TNM staging manual ${ }^{[1]}$ does not contain any staging classification for primary brain tumors, which was omitted from fifth edition onwards. ${ }^{[3]}$ Although primary brain tumors account for $<2 \%$ of all neoplasms ${ }^{[4,5]}$ they are associated with high morbidity and mortality. According to American Cancer Society ${ }^{[5]}$ estimates, 20,500 new patients were 
expected to be diagnosed with primary malignant central nervous system (CNS) tumors with estimated deaths of 12,740 in the year 2007 in the United States of America. Primary brain tumors are the leading cause of death from childhood cancers and the second leading cause of cancer-related deaths in young adult males in the Western world. ${ }^{[5,6]}$ In the absence of a recommended and widely accepted staging system, prognostication is variably based on clinicopathologic factors as considered relevant to the individual patient and open to the judgment of treating physician(s). There is an urgent need to develop a simple yet pragmatic staging/grouping system for primary brain tumors to guide clinical practice.

\section{Aim}

The aim was to develop a simple, pragmatic, and widely applicable grouping/staging system for primary brain tumors, to guide neurooncologists in clinical practice.

\section{Materials and Methods}

The TNM classification has evolved over the years, mainly to improve prognostic ability and to keep pace with the demands of clinical practice. Changes to the TNM classification were previously derived from a decision-making process based on expert opinions from several national TNM committees based on clinical relevance, evidence of improved prognostic ability, and acceptance by member organizations. ${ }^{[2]}$ A Brain Tumor Subcommittee was formed under the aegis of the Indian National TNM. The subcommittee constituted an expert panel comprising of radiation oncologists, neurosurgeons, pathologists, radiologists, and medical oncologists actively engaged in neurooncology practice. The panel deliberated and discussed issues pertinent to brain tumor staging through formal meetings and informally via E-mail: Exchanges.

\section{Results and Recommendations}

The previous UICC and AJCC TNM staging system for brain tumors ${ }^{[7]}$ has been deleted from the fifth and sixth editions of the staging manual, as it is redundant and not in widespread clinical use. A strict anatomic staging classification such as TNM may not be very relevant to brain tumors as they seldom involve regional lymphatics or metastasize outside the CNS. In addition they encompass a plethora of diverse histologies, and it may be more appropriate to classify them into broad histologic subcategories for staging purposes. Three broad histologic subcategories, viz. gliomas, embryonal CNS tumors, and primary CNS lymphomas were chosen for separate grouping/staging classification. This report gives an overview of staging/ grouping of gliomas.

\section{Gliomas}

Gliomas are the commonest primary CNS neoplasms comprising over $40 \%$ of such tumors in adults. ${ }^{[5,6]}$ Histological grade as defined by the World Health Organization (WHO) system ${ }^{[8]}$ is the most important prognostic factor that determines outcome in tumors of glial origin. Histological grading is an attempt to predict the biological behavior of a neoplasm. The WHO classification for CNS neoplasms, including gliomas, employs a grading scheme that is a 'malignancy scale' rather than a strict histological grading system. Grade I glioma refers to lesions having low proliferative potential with a possibility of cure following surgery alone. Grade II tumors are generally diffusely infiltrative in nature despite having low proliferative indices, with a marked potential for progression over time and subsequent transformation toward a higher grade. Only tumors with gross histological evidence of malignancy, such as nuclear atypia, anaplasia, or mitosis, are categorized as grade III tumors. Tumors with microvascular proliferation and/or necrosis in addition to the aforesaid features of malignancy are categorized as grade IV tumors, and are typically associated with a rapid evolution and fatal outcome. Gliomas can thus be subclassified into low-grade (WHO grades I and II) and high-grade (WHO grades III and IV) gliomas. The optimal management of low-grade gliomas, though somewhat debatable, is maximal safe resection with or without adjuvant radiotherapy. ${ }^{[9]}$ For high-grade gliomas, adjuvant radiotherapy with or without chemotherapy following maximal safe resection has been generally recommended. ${ }^{[10]}$ Recent evidence supports the use of concurrent and adjuvant chemotherapy ${ }^{[11]}$ for grade IV gliomas, i.e. glioblastoma. In the absence of a widely accepted staging system, prognosis is based mostly on clinicopathological factors as considered relevant to the individual patient. However, it may be strongly influenced by the biases or prejudices of the treating physician and/or institutional practice patterns and guidelines.

\section{Prognostic indices}

There have been several attempts to develop composite prognostic indices for both low-as well as high-grade gliomas.

\section{Low-grade gliomas}

Bauman and colleagues ${ }^{[12]}$ were the first to pool three large databases (401 patients) to identify pretreatment factors that could predict overall survival in low-grade gliomas. Multivariate analysis showed age 18-40 years, seizures, Karnofsky performance status (KPS) > 70, and absence of contrast enhancement as independent predictors of survival. Recursive partitioning analysis (RPA) yielded four distinct prognostic groups (I-IV), with statistically different median survivals. 
The European Organization for Research and Treatment of Cancer (EORTC) spearheaded two large randomized controlled trials ${ }^{[13,14]}$ that were designed to investigate the dose (EORTC 22844) and timing of postoperative adjuvant radiotherapy (EORTC 22845) in cerebral lowgrade gliomas. Pignatti et al..$^{[15]}$ combined those two datasets to develop a prognostic score for low-grade gliomas. Multivariate analysis on the construction set of 322 patients showed age $\geq 40$ years, astrocytoma histology, tumor diameter $\geq 6 \mathrm{~cm}$, tumor crossing midline, and presence of neurologic deficits preoperatively as unfavorable prognostic factors for survival. Patients with one or two factors had low-risk disease whereas a higher score indicated high-risk disease. The validity of the model was confirmed in the validation set of 288 patients. In a more recent update of EORTC 22845, it was reconfirmed that early radiotherapy delays progression with no impact on overall survival. ${ }^{[16]}$ In the largest analysis of the patterns of care and long-term survival of patients with supratentorial low-grade gliomas, Claus et al. ${ }^{[17]}$ demonstrated increasing survival times (mean six years) over the years, with a quarter of patients surviving for decades.

\section{High-grade gliomas}

The Medical Research Council (MRC) brain tumor working party formulated a three-tiered prognostic index ${ }^{[18]}$ for high-grade gliomas using age, WHO performance status, extent of resection, and history of seizures as prognostic factors. A cumulative score of $>25$ was defined as poor prognosis (median survival 33 weeks), as compared to a score of $\leq 15$ which was classified as favorable (median survival 80 weeks).

The Radiation Therapy Oncology Group (RTOG) used the RPA for classifying malignant gliomas into six prognostic classe ${ }^{[19]}$ using age, grade, KPS, mental status, symptom duration, extent of resection, neurodeficits, and total radiotherapy dose as prognostic factors based on three RTOG trials. Classes V and VI had a particularly poor outcome with a median survival of 8.9 and 4.6 months, respectively. A modified RPA classification ${ }^{[20]}$ based on 832 patients with GBM enrolled on prospective clinical trials uses age, KPS, site, and extent of resection as factors to subdivide the GBM population into four different prognostic groups - low-risk, low-moderaterisk, moderate-high-risk, and high-risk with a median survival of 132, 71, 63, and 37 weeks, respectively.

However, neither the MRC index nor RPA was incorporated into routine clinical practice due to the complexities. A more recent attempt at developing a pragmatic system for prognosis ${ }^{[21]}$ using age and neurological performance status (NPS) as the only prognostic variables discriminates well between the poor and favorable classes, but needs more robust validation in a larger cohort.

\section{Proposed grouping system for gliomas}

Based on data previously published in the indexed medical literature, certain characteristics are defined as unfavorable [Table 1] for the proposed new classification. Since a strict anatomic system may not be very appropriate, a grouping/staging system is proposed [Table 2] as is prevalent in some pediatric solid tumors. ${ }^{[22,23]}$

Group I: (expected excellent long-term survival)

- $\quad$ Any grade I glioma (excepting brainstem gliomas)

Group II: (expected good long-term survival)

- $\quad$ Grade I brainstem glioma (including unverified focal tectal plate gliomas)

- Grade II with no unfavorable characteristics (age, NPS, multicentricity, biology)

Group III: (expected fair long-term survival)

- Grade II with any unfavorable characteristics (age, NPS, multicentricity, biology)

- $\quad$ Grade III with no unfavorable characteristics

Group IV: (expected poor long-term survival)

- $\quad$ Any grade IV glioma

- Grade III with any unfavorable characteristics (age, NPS, multicentricity, biology)

- Any brainstem glioma except grade I or focal tectal plate glioma

Histological grade as defined by WHO system ${ }^{[8]}$ remains the most important prognostic factors predicting outcome in gliomas, hence, it was kept as the primary variable [Table 1]. Age, NPS, multicentricity, and biological parameters were classified as secondary variables. The cut-off age for defining adverse prognosis in low-grade gliomas was kept at 40 years based on the accumulated evidence on low-grade gliomas. ${ }^{[12-15]}$ For high-grade gliomas, the cut-off was taken as 50 years, as this has

\begin{tabular}{|c|c|}
\hline Characteristics & Definition \\
\hline Age (years) & $\begin{array}{l}<5 \text { and }>50 \text { for high-grade gliomas } \\
<5 \text { and }>40 \text { for low-grade gliomas }\end{array}$ \\
\hline Performance status & $\begin{array}{l}\text { Patient not fully independent in } \\
\text { activities of daily living (NPS 2-4) }\end{array}$ \\
\hline $\begin{array}{l}\text { Multicentricity/gli- } \\
\text { omatosis }\end{array}$ & $\begin{array}{l}\text { Multicentric lesions and/or diffuse } \\
\text { gliomatosis }\end{array}$ \\
\hline $\begin{array}{l}\text { Adverse biological } \\
\text { parameters (optional) }\end{array}$ & $\begin{array}{l}\text { MIB-1 index, angiogenesis markers, } \\
\text { apoptotic index, cytogenetic abnor- } \\
\text { malities, and molecular markers }\end{array}$ \\
\hline
\end{tabular}




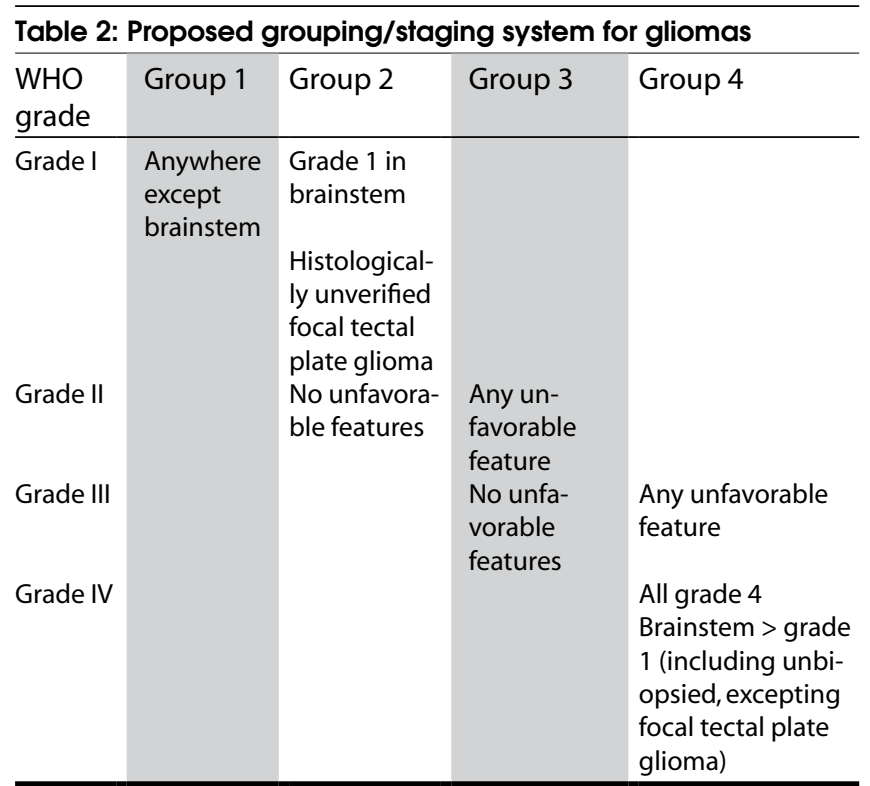

Unfavorable features: Age, NPS, multi-centricity, adverse biology (details in Table 1)

been widely used world over including the RPA-RTOG. ${ }^{[19]}$ There has been a reluctance to use radiation therapy for brain tumors in very young children $(<3$ years of age) due to risk of severe late neurocognitive sequelae. ${ }^{[2,25]}$ In recent times, several cooperative groups have raised this limit and prefer to avoid ${ }^{[26]}$ or at least defer ${ }^{[27]}$ radiotherapy till the age of 5 years. Hence age $<5$ years was also considered an adverse feature due to difficulty in delivering optimal therapy to young children. NPS was considered instead of a general performance scale as it is a more reliable and robust prognostic factor and correlates well with functional independence for brain tumor patients. ${ }^{[21]}$ Apart from conventional adverse histopathological characteristics which determine grade such as hypercellularity, nuclear pleomorphism, mitoses, anaplasia, microvascular/endothelial proliferation, and necrosis, certain other biological parameters not routinely used for grading but increasingly being recognized as reasonable adjuncts to maximize therapeutic benefit (MIB-1 index, angiogenesis markers, apoptotic index, cytogenetic abnormalities, and molecular markers) may be defined for refined classification depending on available infrastructure, expertise, and commitment.

Brainstem gliomas are a heterogeneous group of neoplasms with differing biological behavior and outcome. The most common subtype, diffuse intrinsic pontine glioma $(>75 \%)$, is generally not amenable to biopsy and carries a grave prognosis. The cervicomedullary and the dorsal exophytic subtypes (which can be safely biopsied, sometimes even resected) have better survival. Focal tectal plate gliomas, which are indolent and slow-growing have good long-term survival even without biopsy after cerebrospinal fluid diversion to ameliorate symptoms. Thus, all histologically unverified brainstem gliomas (except focal tectal plate gliomas) are classified in the group with the worst prognosis as they are likely to represent diffuse intrinsic pontine gliomas.

The panel also deliberated on the prognostic significance of several known patients, tumors, and treatment characteristics such as size of lesion, mass effect, midline shift, imaging features, seizures, extent of resection, and total radiation dose. It was a consensus decision to exclude them from the proposed grouping system. This does not reduce the significance of these well-known prognostic factors that need to be considered on a caseto-case basis, but was an attempt to keep the system simple, practical, and widely applicable.

Currently, an important limitation of the proposed system is lack of validation, which the authors propose to overcome by validating it in a large cohort of patients enrolled on their institutional neurooncology database. The proposed system although most pertinent and relevant for pure astrocytomas could be reasonably extended to other gliomas such as oligodendrogliomas or mixed oligoastrocytomas, provided one appreciates their favorable inherent biology and consequent clinical behavior. It is hereby re-emphasized that this simple and pragmatic clinicopathobiological classification is intended to guide neurooncology practice in the community setting and may not be entirely appropriate in the context of prospective clinical trials, for which more elaborate and robust indices already exist.

\section{Conclusion}

In absence of a grouping/staging system for primary brain tumors, prognostication is mostly based on complex composite indices. The proposed grouping/ staging system for gliomas is a simple, pragmatic, and user-friendly tool with a potential to fulfill the objectives of staging classification. This proposed system will require validation in different research and practice settings before it can be recommended for routine clinical practice.

\section{References}

1. Sobin LH, Wittekind CL, editors. TNM classification of malignant tumours. $6^{\text {th }}$ ed. New York: John Wiley and Sons; 2002.

2. Gospodarowicz MK, Miller D, Groome PA, Greene FL, Logan PA, Sobin LH. The process for continuous improvement of the TNM classification. Cancer 2004;100:1-5.

3. Sobin LH, Wittekind CL, editors. TNM classification of malignant tumours. $5^{\text {th }}$ ed. New York: John Wiley and Sons; 1997.

4. Ferlay J, Bray F, Pisani P. GLOBOCAN 2002: Cancer Incidence, Mortality and Prevalence Worldwide, Version 2.0. IARC Cancer Base No. 5. Lyon: IARC Press; 2004.

5. American Cancer Society: Cancer facts and Figures 2007. Atlanta, American Cancer Society, 2007.

6. Central Brain Tumour registry of the United States (CBTRUS). 
Statistical Report 2005-2006. Primary brain tumours in the United States: 1998-2002. Hinsdale, IL.

7. Hermanek P, Sobin LH (editors): TNM classification of malignant tumours, $4^{\text {th }}$ edition, Berlin, Springer-Verlag, 1987.

8. Louis DN, Ohgaki H, Wiestler OD, Cavenee WK, Burger PC, Jouvet A, et al. The 2007 WHO classification of tumours of the central nervous system. Acta Neuropathol 2007;114:97-109.

9. Stupp R, Baumert BG. Promises and controversies in the management of low-grade glioma. Ann Oncol 2003;14:1695-6.

10. Stupp R, Hegi ME, van den Bent MJ, Mason WP, Weller M, Mirimanoff RO, et al. Changing paradigms: An update on the multidisciplinary management of malignant glioma. Oncologist 2006;11:165-80.

11. Stupp R, Mason WP, van den Bent MJ, Weller M, Fisher B, Taphoorn MJ et al. Radiotherapy plus concomitant and adjuvant temozolomide for glioblastoma. N Engl J Med 2005;352:987-96.

12. Bauman G, Lote K, Larson D, Stalpers L, Leighton C, Fisher B et al. Pretreatment factors predict overall survival for patients with low-grade glioma: A recursive partitioning analysis. Int J Radiat Oncol Biol Phys $1999 ; 45: 923-9$

13. Karim AB, Maat B, Hatlevoll R, Menten J, Rutten EH, Thomas DG et al. A randomised trial on dose-response in radiation therapy of low-grade cerebral glioma: European Organization for Research and Treatment of Cancer (EORTC) study 22844. Int J Radiat Oncol Biol Phys 1996;36:549-56.

14. Karim AB, Afra D, Cornu P, Bleehan N, Schraub S, De Witte O et al. Randomised trial of the efficacy of radiotherapy for low-grade glioma in the adult: European Organization for Research and Treatment of Cancer study 22845 with the Medical Research Council study BR04: An interim analysis. Int J Radiat Oncol Biol Phys 2002;52:316-24.

15. Pignatti F, van den Bent M, Curran D, Debruyne C, Sylvester R, Therasse $\mathrm{P}$ et al. Prognostic factors for survival in adult patients with cerebral low-grade glioma. J Clin Oncol 2002;20:2076-84.

16. van den Bent MJ, Afra D, de Witte O, Ben Hassel M, Schraub S, Hoang-Xuan $\mathrm{K}$ et al. Long-term efficacy of early versus delayed radiotherapy for low-grade astrocytoma and oligodendroglioma in adults: The EORTC 22845 randomised trial. Lancet 2005;366:285-90.

17. Claus EB and Black PM: Survival rates and patterns of care for patients diagnosed with supratentorial low-grade gliomas: Data from the SEER program, 1973-2001. Cancer 2006;106:1358-63.

18. MRC Brain Tumour Working Party: Prognostic factors for high-grade malignant gliomas: Development of a prognostic index. J Neurooncol 1990;9:47-55.

19. Curran WJ Jr, Scott CB, Horton J, Nelson JS, Weinstein AS, Fischbach AJ, et al. Recursive partitioning analysis of prognostic factors in three Radiation Therapy Oncology Group malignant glioma trials. J Natl Cancer Inst 1993;85:704-10.

20. Lamborn KR, Chang SM, and Prados MD: Prognostic factors for survival of patients with glioblastoma: Recursive partitioning analysis. Neuro-Oncol 2004;6:227-35.

21. Gupta T, Sarin R: Poor-prognosis high grade gliomas: Evolving an evidence-based standard of care. Lancet Oncol 2002;3:557-64.

22. Raney RB, Anderson JR, Barr FG, Donaldson SS, Pappo AS, Qualman SJ, et al. Rhabdomyosarcoma and undifferentiated sarcoma in the first two decades of life: A selective review of Intergroup Rhabdomyosarcoma study experience and rationale for Intergroup Rhabdomyosarcoma Study V. J Pediatr Hematol Oncol 2001;23:215-20.

23. Abramson DH. Retinoblastoma: Diagnosis and management. CA Cancer J Clin 1982;32:130-40.

24. Duffner PK, Horowitz ME, Krischer JP, Burger PC, Cohen ME, Sanford RA et al. The treatment of malignant brain tumours in infants and very young children: An update of the Pediatric Oncology Group experience. Neuro-Oncol 1999;1:152-61.

25. Fouladi M, Gilger E, Kocak M, Wallace D, Buchanan G, Reeves C et al. Intellectual and functional outcome of children 3 years old or younger who have CNS malignancies. J Clin Oncol 2005;23:7152-60.

26. Grundy RG, Wilne SA, Weston CL, Robinson K, Lashford LS, Ironside J et al. Primary postoperative chemotherapy without radiotherapy for intracranial ependymoma in children: The UKCCSG/SIOP prospective study. Lancet Oncol 2007;8:696-705.

27. Dufour C, Grill J, Lellouch-Tubiana A, Puget S, Chastagner P, et al. High-grade glioma in children under 5 years of age: A chemotherapy only approach with BBSFOP protocol. Eur J Cancer 2006;42:2939-45.

Accepted on 15-02-2009

Source of Support: Nil, Conflict of Interest: None declared. 